\title{
Experimental investigation on the aerodynamic loads and wake flow features of a low aspect-ratio circular cylinder
}

\author{
G.V. Iungo*, L.M. Pii, G. Buresti \\ University of Pisa, Department of Aerospace Engineering, via Caruso 8, 56122 Pisa, Italy
}

\section{A R T I C L E I N F O}

\section{Article history:}

Received 20 December 2010

Accepted 13 November 2011

Available online 5 December 2011

\section{Keywords:}

Bluff body aerodynamic

Circular cylinder

Wakes

Flow fluctuations

Vorticity dynamics

\begin{abstract}
A B S T R A C T
An experimental investigation on the flow features of the wake generated from a circular cylinder with finite height and placed vertically on a plane is presented. Through force measurements the mean drag coefficient is found to be roughly invariant by varying Reynolds number in a range between $6 \times 10^{4}$ and $11 \times 10^{4}$. As for the fluctuating forces, a dominant spectral component is clearly detected for the signals of the cross-flow force. A spectral contribution with roughly the same Strouhal number is detected from velocity signals acquired, through hot-wire anemometry, in proximity to the lateral wake boundary; its energy is found to decrease by moving the probe away from the wake and upwards. Simultaneous velocity measurements showed that these fluctuations can confidently be ascribed to an alternate vortex shedding. Subsequently, dynamic measurements of the pressure field over the lateral surface and the free-end of the model were carried out, which highlight that the spectral component connected to vortex shedding is found over the lateral surface, with maximum energy at an azimuthal position just before the separation of the shear layers. The fluctuating energy connected to vortex shedding decreases by moving towards regions immersed in the separated wake, and with increasing vertical coordinate; as a matter of fact, above about half model height an evident energy peak cannot be detected anymore. This feature highlights that a regular alternate vortex shedding occurs only for the lower half-span of the model and that the remaining part is dominated by the upwash generated by the flow passing over the free-end. From the spectral analysis of the pressure measurements carried out over the model free-end no evidence of the presence of the spectral component connected to the alternate vortex shedding is found, as expected. However, a significant fluctuating energy is observed at lower dominating frequencies.
\end{abstract}

(c) 2011 Elsevier Ltd. All rights reserved.

\section{Introduction}

The wakes of finite circular cylinders in cross-wind are dominated by the velocity fluctuations induced by the alternate vortex shedding from the body sides, as documented by Farivar (1981), Ayoub and Karamcheti (1982), Fox et al. (1993), Bearman (2011), Griffith et al. (2011), Suthon and Dalton (2011) and Visscher et al. (2011), in which the presence of clear vortex shedding from most of the cylinder span, with frequencies of the same order as those typical of twodimensional flow, was found for models with aspect-ratio $h / d>10$ (where $h$ is the cylinder height and $d$ its diameter).

\footnotetext{
* Corresponding author. Tel.: +41 2169 33096; fax: +41216936135.

E-mail addresses: giacomo.iungo@ing.unipi.it, valerio.iungo@epfl.ch (G.V. Iungo).
} 
However, a decrease of the frequency was found in a zone approaching the upper end of the cylinders, probably due to an increase in the formation length of the shed vortices. The relative extent of this zone, which is likely to be a cell with lower-frequency shedding, increases with decreasing aspect-ratio, and for $h / d<5$ the vortex shedding from the whole body takes place at a frequency that is lower than in the corresponding two-dimensional case (Sakamoto and Arie, 1983).

Moreover, further fluctuations connected with the dynamics of the vorticity structures originated by the flow passing over the body free-end may appear in the near wake. In effect, the presence of a free-end gives rise to an intense local flow, which passes over the body tip and is deflected within the separated wake by the low pressures that are present in that region. This flow varies the width of the wake and the pressure field with respect to the two-dimensional condition, and may interact with the flow rounding the sides of the body, thus producing a significant interference with the regular vortex shedding mechanism.

A couple of counter-rotating streamwise vortices generated over the free-end of a model with $h / d=5$ were visualized by Park and Lee (2000) through a particle tracer technique and light sheet. These vortices, similar to the tip-vortices formed along the leading edge of a delta wing, bend into the wake after detaching from the free-end and interact with the vortices alternatively shed from the lateral surface of the model. An analogous couple of counter-rotating axial vortices generated over the free-end of a model with $h / d=3$ were found in Buresti and Lombardi (2003).

Oil and smoke-laser sheet visualizations were carried out in Roh and Park (2003) for circular cylinders with aspectratios 1.25 and 4.25 . It was found that, apart from the well-known couple of side counter-rotating tip-vortices detached from the free-end, another couple of counter-rotating vortices are present for inner positions than the former ones. For each side of the wake these vortices were found to be counter-rotating with respect to the side tip-vortices. From the oil flow visualizations it is observed that these additional vortices are generated over the free-end in locations where the incoming freestream interacts with the reversed flow present in the recirculating region generated over the free-end. This feature is also confirmed by the oil flow visualizations performed by Pattenden et al. (2005).

The influence of the free-end vorticity structures on the two-dimensional vortex shedding along the span of circular cylinders is, as expected, strictly related to the model aspect-ratio, $h / d$. In Farivar (1981), for finite cylinders with aspectratio ranging from 10 to 12.5 and tested with a freestream velocity, $U_{\infty}$, corresponding to $\operatorname{Re}=U_{\infty} \times d / v \approx 0.7 \times 10^{5}$, a cellular shedding was found. In the wake cell-like structures the Strouhal number, St $=f \times d / U_{\infty}$, was found to be constant within each cell, but varied across cells. Along the model span three cells with a constant Strouhal number were detected by these authors through hot-wire signals and surface pressure measurements. The lowest Strouhal number,

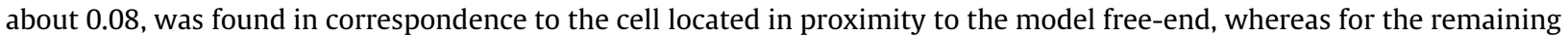
cells the associated Strouhal number increases by moving towards the model base.

A lower frequency, about half of the one corresponding to vortex shedding, was also found by Ayoub and Karamcheti ( 1982 ) for a model with $h / d \approx 12$ and tested at $\operatorname{Re} \approx 0.85 \times 10^{5}$. A cellular vortex shedding with a lower frequency of St $\approx 0.07$ was observed in Khalak and Williamson (1996) for cylinders with aspect-ratio 8.5 and 10 . A significant spectral component corresponding to a Strouhal number of 0.08 was found in Luo et al. (1996) at a height of $0.95 \mathrm{~h}$ for a model with aspect-ratio 8; however, no wake cellular structure was detected.

A characterization of the cellular structure of the wake of a circular cylinder with $h / d=62.5$ was carried out by Stocks et al. (1999) through hot-wire anemometry. A dominant central cell, corresponding to the main vortex shedding mode, surrounded by two end-cells characterized by a lower shedding frequency, was detected just downstream of the model. A third cell is generated by the nonlinear interaction of these two cellular modes, which is characterized by St $\approx 0.03$. This spectral component propagates downstream whilst the higher frequencies are filtered out.

A lower frequency at St $\approx 0.07$ was found in Kitagawa et al. (1999) and was ascribed to the dynamics of the tip-vortices generated over the free-end of a model with $h / d=25$. In Kitagawa et al. (2002) it was then highlighted that this spectral component was due to a non-alternate oscillation of the axial vorticity structures generated by the flow passing over the free-end, even if this motion was found to be highly irregular.

In Park and Lee (2000) circular cylinders with aspect-ratio ranging from 6 to 13 were tested at $\operatorname{Re} \approx 2 \times 10^{5}$ and a low-frequency component at $\mathrm{St} \approx 0.07$ was detected with an energy content that increases with increasing streamwise distance from the model and by moving from the model base towards the free-end, but which is reduced with decreasing aspect-ratio. From simultaneous measurements carried out in proximity to the lateral wake boundary, at symmetrical locations with respect to the symmetry plane, no phase difference was found on the two wake sides, which suggests a non-alternate oscillation of the vorticity structures present in the upper-wake. In Park and Lee (2002) it was highlighted that this spectral component is clearly detectable in the near-wake; however, proceeding downstream it is difficult to detect it because the tipvortices gradually bend downwards into the wake. Furthermore, the low-frequency component was observed to be present with the same frequency for different boundary layer heights and with different free-end shapes (see Park and Lee, 2004); the latter influence only the evolution and the energy of the spectral component, but not its frequency.

In the present work an experimental investigation on the wake flow generated from a circular cylinder placed vertically on a plane is described. This work aims to achieve a deep characterization of the wake morphology, of the dynamics connected with the wake vorticity structures, and to evaluate their effects on the mean and fluctuating loads. The analyzed model is characterized by $h / d=3$, which is a sufficiently low aspect-ratio in order to ensure a strong three-dimensional effect of the flow passing over the free-end on vortex shedding, as reported in Sakamoto and Arie (1983), i.e. vortex shedding from the cylinder takes place at a lower frequency than in the corresponding two-dimensional case. Moreover, these authors also found that for models with $h / d<2$ the vortex shedding switches from a Karman-type to the arch-type 
vortex. Therefore, although the considered model with $h / d=3$ produces a strong three-dimensional effect on vortex shedding, it is still characterized by a Karman-type wake.

The paper is organized as follows. The facility and all the measurement procedures are described in Section 2 . The force measurements are described in Section 3, while the results obtained from hot-wire measurements are reported in Section 4. The statistical and spectral characterization of the pressure measurements carried out over the lateral surface and the free-end of the model are presented in Section 5. Finally, some conclusions are drawn in Section 6.

\section{Experimental set-up and procedures}

The tests were carried out in the closed-return, subsonic wind tunnel of the Department of Aerospace Engineering of the University of Pisa, which is characterized by a circular open test section $1.1 \mathrm{~m}$ in diameter and $1.42 \mathrm{~m}$ in length, and freestream turbulence level of $0.9 \%$. The general set-up of the tests is shown in Fig. 1.

The model is a circular cylinder with aspect-ratio $h / d=3$ and diameter $d=60 \mathrm{~mm}$. As already mentioned, the selected aspect-ratio is sufficiently small to guarantee a significant three-dimensional effect of the flow passing over the model free-end on vortex shedding, but still producing a Karman-type wake (see Sakamoto and Arie, 1983). The model was placed vertically on a horizontal plane and connected to a six-component strain-gage balance supported by a rotatable base placed underneath, so that it could easily be rotated around the model axis. A NACA 0018 fairing was used to shield the balance and the relevant support. The boundary layer thickness in proximity of the model is equal to $9 \%$ of the model height, ensuring a negligible influence on the vortex shedding frequency and on the free-end flow (see Sakamoto and Arie, 1983). The used frame of reference, whose origin is positioned in correspondence to the intersection between the model axis and its base, is shown in Fig. 1 . The $x$-axis was chosen in the freestream direction and the $z$-axis is coincident with the model axis, positive from the model base to the tip. The $y$ direction was consequently defined, producing a right-handed frame of reference.

A differential pressure transducer SETRA model 239 was used to measure the wind-tunnel dynamic pressure, and the static temperature was measured through a Tecnopound Pt100 probe. All the signals were acquired with a D.A.Q. NI 4472, whilst the traversing apparatus and the selection of the pressure scanner ports were driven by a digital D.A.Q. NI 6503. All the tests were carried out at a freestream velocity between 15 and $28 \mathrm{~m} / \mathrm{s}$ corresponding to a Reynolds number between $6 \times 10^{4}$ and $11 \times 10^{4}$.

As for the force measurements, the six signals from the balance, each comprising 16384 samples, were simultaneously acquired at a sampling rate of $1 \mathrm{kHz}$, and were processed by means of the balance calibration matrix to obtain the time histories of the load components acting on the model. The maximum errors of the balance measurements were previously estimated to be of the order of $1.5 \%$.

Velocity measurements were performed with single-component hot-wire anemometry. The used probes were Dantec type 55, connected to an IFA AN 1003 A.A. Lab System test module. A sampling frequency of $2 \mathrm{kHz}$ and a time-length of $33 \mathrm{~s}$ were chosen after extensive preliminary tests.

Pressure measurements were carried out using two Pressure Systems ESP-16HD electronic pressure scanners, each with 16 ports and transducers, which were previously calibrated. The pressure taps on the model surface, about 0.5 mm in diameter, were connected to the respective ports through $400 \mathrm{~mm}$ long plastic tubes, assuring that the highest frequency of interest (viz. that of vortex shedding) could adequately be described. From each port, signals comprising $2^{15}$ samples were acquired with a sampling rate of $1 \mathrm{kHz}$. Fifteen taps were placed along one generatrix of the lateral surface, which is rotated at different azimuthal angles, $\theta$, from the flow stagnation area at $\theta=0^{\circ}$ to $\theta=180^{\circ}$. Pressure measurements were also carried out over the model free-end through 65 taps evenly spaced with an azimuthal step of $22.5^{\circ}$ and a radial distance of about $6 \mathrm{~mm}$.

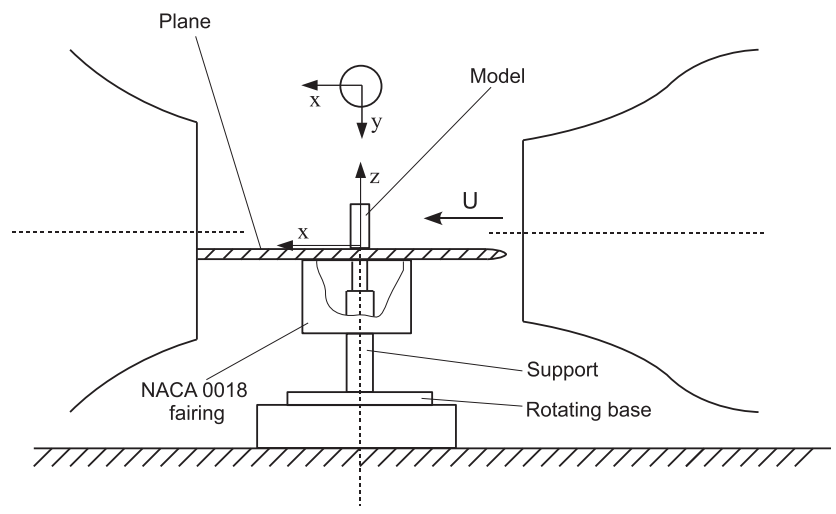

Fig. 1. Experimental layout. 
The measurements were analyzed using conventional Fourier techniques and procedures based on the wavelet and Hilbert transforms. In particular, the complex Morlet wavelet, defined as $\psi(t)=e^{i \omega_{0} t} e^{-t^{2} / 2}$, was used. The spectra described in the following were obtained by integration in time of the wavelet maps obtained by using a central frequency $\omega_{0}=6 \pi$, in order to enhance the frequency resolution. Finally, the procedure based on wavelet filtering and Hilbert transform, described in Buresti et al. (2004), was used in order to extract the main spectral contributions from the signals.

\section{Force measurements}

Force measurements were carried out for several freestream velocity values between 15 and $28 \mathrm{~m} / \mathrm{s}$, and six measurements were performed for each velocity. Forces are represented through their respective coefficients, using as reference values the freestream dynamic pressure and the surface $h \times d$. In Table 1 the statistics related to the drag coefficient, $C_{x}$, are reported. The mean value of $C_{x}$ is evaluated to be about 0.81 with a standard deviation of about $10 \%$ of the mean value. Furthermore, the mean value of $C_{x}$ is found to be roughly invariant with varying Reynolds number, as expected, because all the tested freestream conditions correspond to a subcritical regime, i.e. with a laminar flow separation from the lateral model surface. The obtained mean drag coefficient is in good agreement with the data reported in E.S.D.U. (2005) for a circular cylinder with $h / d=3$ and smooth surface; in that report an empirical law for surface roughness correction is also proposed.

The mean values of the drag coefficient obtained for a circular cylinder with $h / d=3$ in different experimental works are reported in Table 2. In that table the tested Reynolds number, the boundary layer thickness on the plane, $\delta$, and the freestream turbulence level, $T u$, are also reported. The corresponding values for the present work are $\delta / h=0.09$ and $\mathrm{Tu}=0.9 \%$.

It is evident that the mean $C_{x}$ evaluated from the present experimental campaign is higher than all the remaining ones reported in Table 2. This discrepancy may be ascribed to differences either in Reynolds number or in boundary layer thickness, $\delta$. With respect to the result of Okamoto and Yagita (1973), in the present work a slightly higher $C_{x}$ is found, in spite of a comparable boundary layer thickness, which could be an effect of a different Reynolds number. Indeed, from E.S.D.U. (2005) a lower drag coefficient is reported for $\operatorname{Re}=1.3 \times 10^{4}$ with respect to conditions with $\operatorname{Re}>3 \times 10^{4}$.

For the remaining tests performed roughly with the same Reynolds number, a drag reduction by increasing the ratio between the boundary layer thickness and the model height, $\delta / h$, is found, since in all cases the drag coefficients are evaluated with reference to the velocity outside the boundary layer. This trend was also confirmed in Taniguchi et al. (1981), where an empirical law to evaluate the mean drag coefficient as a function of $\delta / h$ was proposed:

$$
C_{x}=a\left(\frac{\delta}{h}\right)^{-b},
$$

where $a$ and $b$ are calculated as follows:

$$
\left.\begin{array}{l}
a=0.617 \\
b=0.157
\end{array}\right\} \text { for } \frac{\delta}{H}<0.91 \text {. }
$$

Table 1

Statistical characterization of the drag coefficient, $C_{x}$, for different freestream velocities.

\begin{tabular}{lll}
\hline Re & $C_{x}$ mean & $C_{x}$ std \\
\hline $6.0 \times 10^{4}$ & 0.82 & 0.07 \\
$7.2 \times 10^{4}$ & 0.79 & 0.07 \\
$8.0 \times 10^{4}$ & 0.81 & 0.07 \\
$9.3 \times 10^{4}$ & 0.81 & 0.08 \\
$10.1 \times 10^{4}$ & 0.80 & 0.09 \\
$11.3 \times 10^{4}$ & 0.81 & 0.09 \\
Mean & 0.81 & 0.08 \\
\hline
\end{tabular}

Table 2

Mean drag coefficient, $C_{x}$, evaluated from different experimental works for a circular cylinder with $h / d=3$. (I: calculated through numerical integration of

\begin{tabular}{|c|c|c|c|c|c|}
\hline Authors & $C_{x}$ & $\operatorname{Re}$ & $\delta / h$ & $\mathrm{Tu}(\%)$ & Notes \\
\hline Farivar (1981) & 0.63 & 70000 & - & 0.4 & \\
\hline Sumner et al. (2004) & 0.61 & 60000 & 0.87 & 0.6 & \\
\hline Taniguchi et al. (1981) & 0.64 & $30000-60000$ & 0.83 & 0.3 & $I$ \\
\hline Sakamoto and Oiwake (1984) & 0.68 & 60000 & 0.67 & - & \\
\hline Okamoto and Yagita (1973) & 0.75 & 13000 & 0.05 & 0.45 & $I, L$ \\
\hline
\end{tabular}
pressure measurements; $L$ : laminar boundary layer on plate.) 


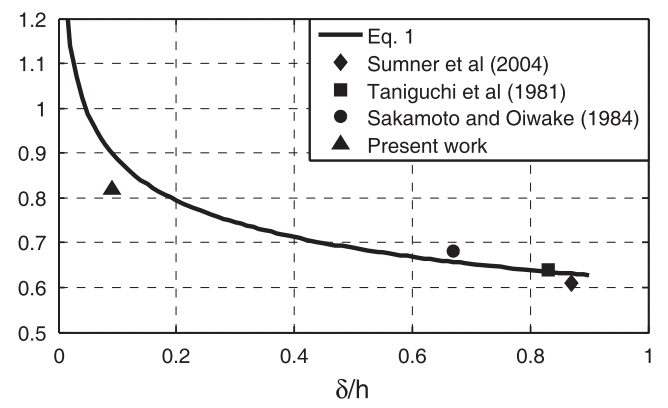

Fig. 2. Comparison between the empirical law proposed by Taniguchi et al. (1981) to predict the mean $C_{x}$ of a circular cylinder with finite aspect-ratio as a function of $\delta / h$ and results of several experimental works.

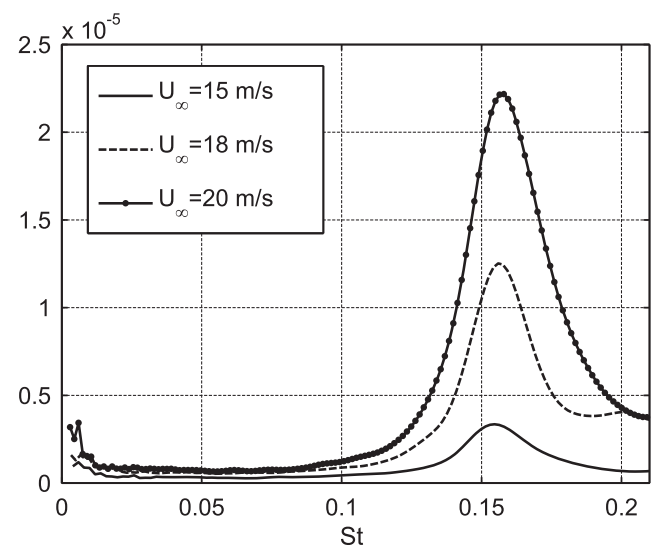

Fig. 3. Wavelet spectra of the cross-flow force for different freestream velocities.

A good agreement is generally observed in Fig. 2 between the mean drag coefficients reported in Table 2 and the empirical law (Eq. (1)) proposed by Taniguchi et al. (1981).

As for the cross-flow force, its mean value is found to be roughly zero due to the model symmetry. At variance with $C_{x}$, for which a roughly constant standard deviation has been found for different freestream velocities, the standard deviation of $C_{y}$ increases monotonically with increasing $U_{\infty}$, most probably due to the increased strength of the vorticity structures detaching from the lateral surface of the model.

From the spectral analysis of the force signals, a spectral component at the vortex shedding frequency may be expected for the cross-flow force, together with a smaller contribution at the double of the vortex shedding frequency for the drag force. However, no dominant spectral components were detected for the drag force, probably due to the small energy of its fluctuations.

As for the cross-flow force, a dominant spectral component was generally observed, as may be appreciated from the wavelet spectra in Fig. 3. By performing a spectral component extraction, through the wavelet-Hilbert technique described in Buresti et al. (2004), a mean Strouhal number of 0.156 is found with fluctuations of about $3 \%$ of its mean value. This spectral component can confidently be ascribed to the alternate vortex shedding, as will be shown in the following section.

Force measurements of the same model and at the same Reynolds number of the present experimental campaign were performed by Sakamoto and Oiwake (1984), but they found from the cross-flow force signals a dominant spectral component with $S t \approx 0.14$. This discrepancy is most probably due to the different boundary layer thickness, $\delta / h \approx 0.67$ for Sakamoto and Oiwake (1984) and $\delta / h \approx 0.09$ for the present work. As matter of fact, an invariant Strouhal number was found by Sakamoto and Arie (1983) by varying the boundary layer thickness for $\delta / h<0.3$, whereas for higher $\delta / h$ it decreases with increasing $\delta$.

\section{Hot-wire measurements}

Hot-wire measurements were carried out in order to characterize the wake morphology and to detect the main spectral components due to the oscillations of the wake vorticity structures. All the tests were performed with a freestream velocity of $25 \mathrm{~m} / \mathrm{s}$ and inside a space domain extending to a downstream distance of 5.5d, for heights between $0.3 \mathrm{~h}$ and $1.1 \mathrm{~h}$. 
In Fig. 4 the mean value and the standard deviation of the velocity measurements carried out at $x / d=2, x / d=3$ and $x / d=4.5$ and different heights are reported. An increase of the wake width with reducing vertical position is apparent from the mean values for all the streamwise distances. This feature is also confirmed from the plots of the standard deviation for which the wake width can be considered to be proportional to the transversal distance from the peaks. Furthermore, a progressive widening and lowering of the wake is observed by proceeding downstream. All these features involving changing of the wake width and height are most probably due to the flow passing over the model free-end that generates a significant downwash on the upper-part of the wake.
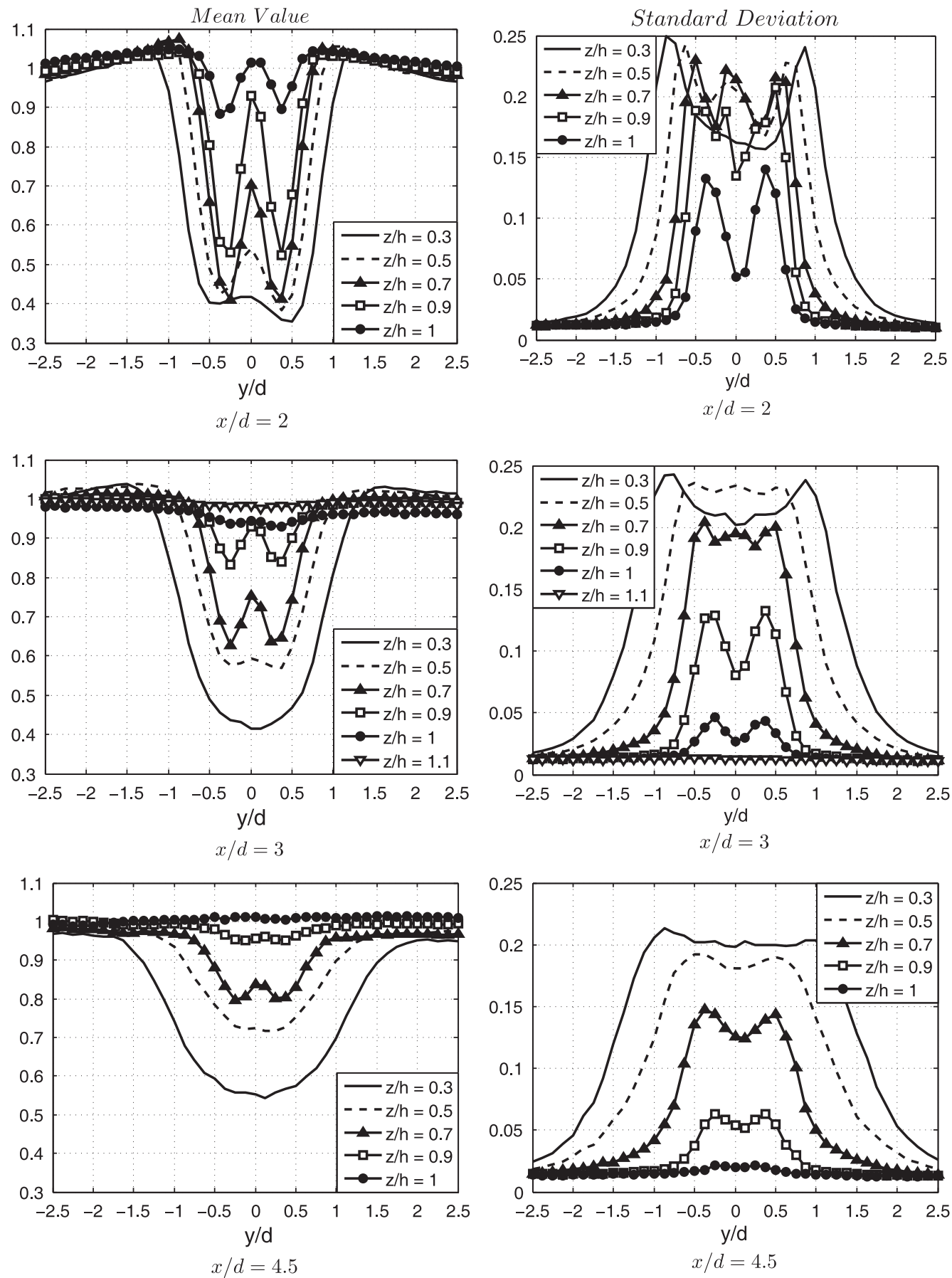

Fig. 4. Statistics of the hot-wire measurements for different streamwise distances and heights. On the left-hand side the mean value and on the righthand side the standard deviation. 
It is interesting to observe from the plots of the mean value that in proximity to the wake symmetry plane, $y / d=0$, a local peak of this quantity is present for different traverses, which suggests the presence of an increased modulus of the velocity vector. This feature is particularly enhanced for the higher vertical positions and for the streamwise location $x / d=2$. This feature is most probably due to the downwash acting on the upper-wake generated by the shear layers detaching over the model free-end, and to the velocity field induced by the couple of counter-rotating axial vortices detaching over the free-end. Moreover, for the traverse carried out at $x / d=2$ and $z / h=1$ a velocity roughly equal to the freestream is measured for $y / d=0$ indicating that a significant lowering of the wake has already occurred in proximity to the wake symmetry plane.

From the spectral analysis of the velocity signals, a dominant spectral component at $\mathrm{St} \approx 0.15$ is found, as can be seen in Fig. 5 in which wavelet spectra of signals acquired at different downstream distances and heights are reported. This spectral component is particularly evident in proximity to the lateral wake boundary and its energy decreases moving horizontally the probe away from the wake. Moreover, this component is highly dominant for $z / h<0.7$, with fluctuating energy that decreases with increasing vertical position.

Maps of the fluctuating energy, evaluated through wavelet transform, of velocity signals acquired at different streamwise locations and at $z / h=0.3$ are reported in Fig. 6. At $x / d=2$ the fluctuating energy corresponding to St $\approx 0.15$ is well concentrated at the lateral wake boundary. By proceeding downstream, at $x / d=3$ this contribution is observed also for inner regions located inside the wake, while further downstream at $x / d=4.5$ it is slightly decreased and more diffuse.

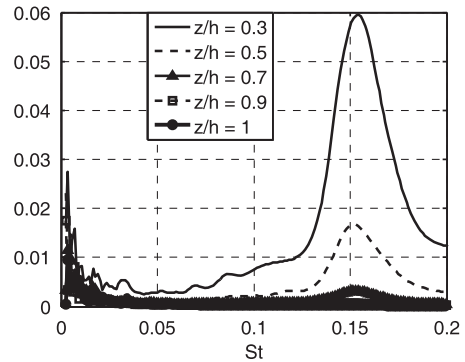

(a)

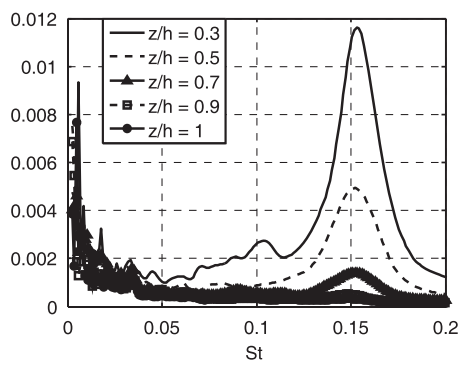

(b)

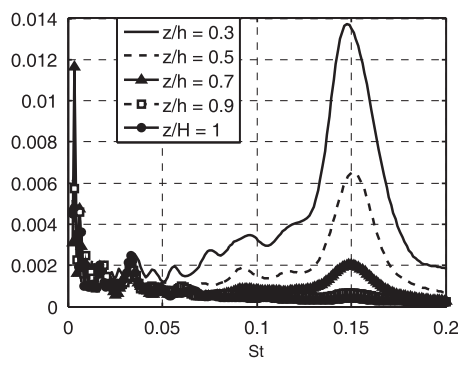

(c)

Fig. 5. Wavelet spectra of hot-wire signals acquired at different locations and for different heights: (a) $x / d=2, y / d=-1.4$; $(b) x / d=3, y / d=-2.1$; (c) $x / d=4.5, y / d=-2.4$.
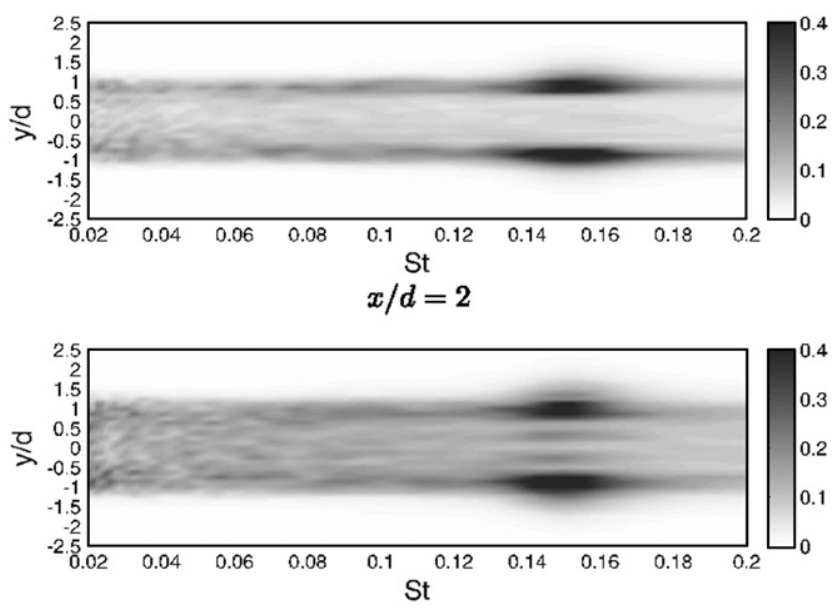

$x / d=3$

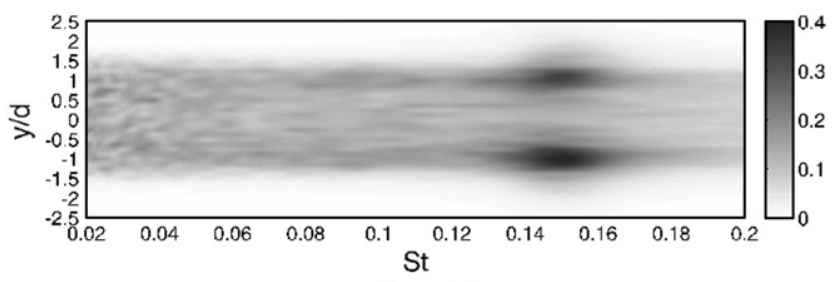

$x / d=4.5$

Fig. 6. Maps of the wavelet fluctuating energy for different traverses carried out at $z / h=0.3$ and different streamwise locations. 
The spectral component of interest is then extracted through the technique based on the wavelet and Hilbert transforms to obtain a time-frequency characterization, and all the related statistics are reported in Table 3 . A mean value of $\mathrm{St}=0.151$ is evaluated and the statistics are definitely comparable to what has been evaluated through the force measurements. From other experimental works slightly different vortex shedding frequencies were found for a circular cylinder with $h / d=3$ through hot-wire measurements, as e.g. St=0.145 in Sakamoto and Arie (1983). Moreover, other authors like Farivar (1981), Okamoto and Yagita (1973), Budair et al. (1991), Okamoto and Sunabashiri (1992), Fröhlich and Rodi (2004) did not found a dominant spectral contribution related to vortex shedding. This evident disagreement between the results of different experimental works is probably due to the different values of the boundary layer thickness as percentage of the model height and of the freestream turbulence level.

Finally, simultaneous measurements carried out at symmetrical locations with respect to the wake symmetry plane were carried out. For each couple of points three measurements were performed. The objective of these tests is to investigate on the phase-shift between two spectral components related to St $\approx 0.15$ extracted from simultaneous acquired velocity signals. The components filtering is performed through wavelet transform and their cross-correlation is then calculated through the Hilbert local cross-correlation coefficient, HLCC, (see Buresti et al., 2004). HLCC is based on the definition of a "cross-analytical signal" using the Hilbert transform, and provides the time variation of the crosscorrelation between two signals having similar frequencies: when the coefficient HLCC $=1$ the phase-shift between two spectral components is negligible, whereas when HLCC $=-1$ the phase-shift is $180^{\circ}$.

In Fig. 7(a) time-histories of the spectral components of St $\approx 0.15$ are shown; they are extracted from velocity signals simultaneously acquired at $x / d=2, y / d= \pm 0.75$ and $z / h=0.3$ through a bandpass filer with an amplitude of $20 \%$ of the central frequency. In Fig. 7(b) their local frequency is also reported. It is apparent that these components are characterized by a phase-shift of almost $180^{\circ}$.

The statistics of the cross-correlation of the spectral components of interest extracted from signals acquired simultaneously at symmetrical locations are reported in Table 4 . The statistics are calculated as average of three different measurements. The highly negative median value of HLCC that was found indicates that the fluctuations at St $\approx 0.15$ are observed alternatively on the two sides of the wake; therefore, this spectral component can confidently be ascribed to an alternate vortex shedding.

Table 3

Statistical characterization of the spectral contribution of St $\approx 0.15$ from hot-wire measurements (std $=$ standard deviation).

\begin{tabular}{|c|c|c|c|c|c|}
\hline$x / d$ & $z / h$ & St mean & St std & St skewness & St kurtosis \\
\hline 2 & 0.3 & 0.153 & 0.005 & -0.050 & 3.755 \\
\hline 2 & 0.5 & 0.152 & 0.005 & -0.040 & 3.949 \\
\hline 2 & 0.7 & 0.153 & 0.005 & 0.021 & 4.084 \\
\hline 3 & 0.3 & 0.150 & 0.005 & -0.018 & 3.603 \\
\hline 3 & 0.5 & 0.151 & 0.005 & 0.008 & 3.887 \\
\hline 3 & 0.7 & 0.151 & 0.005 & 0.002 & 3.919 \\
\hline 4.5 & 0.3 & 0.149 & 0.005 & 0.058 & 3.791 \\
\hline 4.5 & 0.5 & 0.150 & 0.005 & -0.020 & 3.665 \\
\hline 4.5 & 0.7 & 0.150 & 0.005 & 0.032 & 3.782 \\
\hline Mean & & 0.151 & 0.005 & 0.000 & 3.826 \\
\hline
\end{tabular}

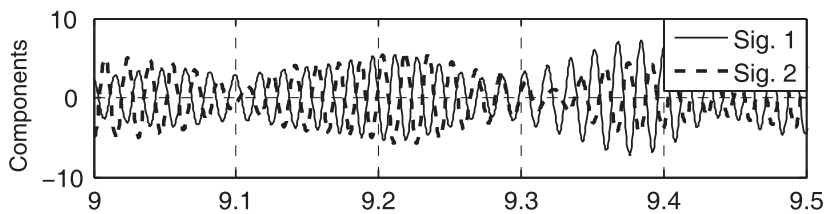

(a)

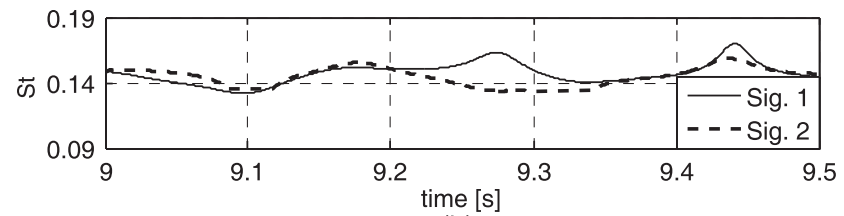

(b)

Fig. 7. Cross-analysis of the spectral contribution related to $\mathrm{St} \approx 0.15$ extracted from the velocity signals acquired at $x / d=2, y / d= \pm 0.75, z / h=0.3$ : (a) extracted components; (b) local frequency. 
Besides the vortex shedding frequency, in the wake flow several authors (see e.g. Ayoub and Karamcheti, 1982; Farivar, 1981; Khalak and Williamson, 1996; Luo et al., 1996) detected another spectral component in the wake flow, which is characterized by a lower frequency and is connected with the dynamics of vorticity structures originated over the model free-end. Conversely, for the present velocity measurements a low spectral component is not found, which is most probably due to the low aspect-ratio, $h / d=3$, of the analyzed model. As a matter of fact, in Park and Lee (2000) circular cylinders with aspect-ratio ranging from 6 to 13 were tested and energy of the low-frequency component was found to be reduced with decreasing aspect-ratio.

\section{Pressure measurements}

Pressure measurements over the lateral surface and the free-end of the model were carried out in order to investigate on the mean and fluctuating pressure field acting on the model. Along one generatrix of the model 15 taps were placed and the tests were performed by rotating the model, from the orientation for which the taps were positioned in correspondence to the stagnation area, viz. $\theta=0^{\circ}$, to $\theta=180^{\circ}$. Different azimuthal steps were used, considering the variation of the pressure gradient by varying the azimuthal position, $\theta$ : an azimuthal step of $1^{\circ}$ was used for the range $0^{\circ} \leq \theta \leq 5^{\circ}$, of $5^{\circ}$ for the range $5^{\circ} \leq \theta \leq 90^{\circ}$ and of $10^{\circ}$ for the range $90^{\circ} \leq \theta \leq 180^{\circ}$. As for the free-end, the measurements were carried out through 65 taps evenly spaced with an azimuthal step of $22.5^{\circ}$ and positioned on four different circumferences placed at a radial distance from the model axis of $6,12.5,19$ and $25 \mathrm{~mm}$. The tests were performed at freestream velocities of 15,20 and $25 \mathrm{~m} / \mathrm{s}$. The measured pressure is expressed through the non-dimensional coefficient, $C_{p}$.

The mean pressures measured at different vertical locations over the lateral surface of the model are shown in Fig. 8(a) as a function of the azimuthal coordinate, $\theta$. As can be seen, a continuous reduction of $C_{p}$ is observed by moving from the flow stagnation area at $\theta=0^{\circ}$ to $\theta \approx 65^{\circ}$, due to the increasing of the flow velocity over the model surface. For higher values of $\theta$ a positive gradient of $C_{p}$ is observed up to $\theta \approx 80^{\circ}$ and for that angle the flow can be considered completely separated over the lateral surface, for all the tested vertical positions. The negative peak of $C_{p}$ is observed at $\theta \approx 65^{\circ}$, but with a modulus that increases with increasing $z / h$; this feature is most probably due to the three-dimensional flow generated from the presence of a free-end, which involves a significant flow passing over the free-end and, thus, an upwash over the lateral surface. Obviously, this phenomenon is more intense in proximity to the model free-end. The measured mean pressure field is generally in good agreement with the one reported in Okamoto and Sunabashiri (1992).

In Fig. 8(b) the standard deviation as a function of $\theta$ and for different values of $z / h$ is reported. Pressure fluctuations decrease by moving from $\theta=0^{\circ}$ up to $\theta \approx 35^{\circ}$. For higher values of $\theta$ it starts increasing up to $\theta \approx 65^{\circ}$, in correspondence to the negative peak of $C_{p}$, and then it decreases again up to the location of the flow separation $\left(\theta \approx 80^{\circ}\right)$, after which it remains fairly invariant for locations into the separated wake.

The mean pressure field acting on the lateral surface is also reported in Fig. 9 as a function of $z / h$ and for the different azimuthal locations, $\theta$. Starting from the curve corresponding to $\theta=0^{\circ}$, a lower $C_{p}$ is observed in correspondence to the model free-end and to the model base. As for the former, this feature can be ascribed, as already mentioned, to the upwash

Table 4

Statistical characterization of the Hilbert local cross-correlation coefficient between simultaneously acquired spectral components related to St $\approx 0.15$.

\begin{tabular}{llccc}
\hline$x / d$ & $y / d$ & $z / h$ & HLCC mean & HLCC median \\
\hline 2 & \pm 0.75 & 0.3 & -0.843 & -0.940 \\
3 & \pm 1.5 & 0.3 & -0.892 & -0.954 \\
Mean & & & -0.867 & -0.947 \\
\hline
\end{tabular}

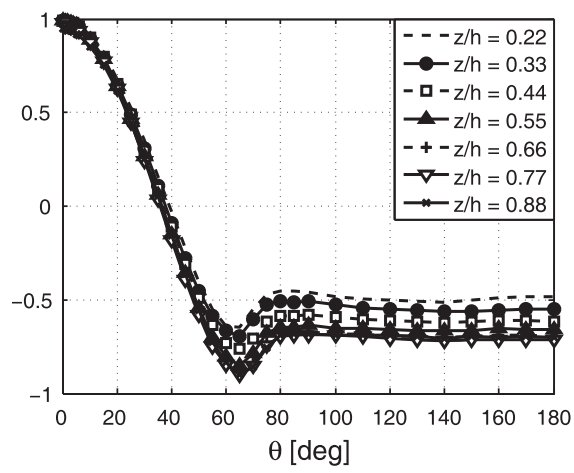

(a)

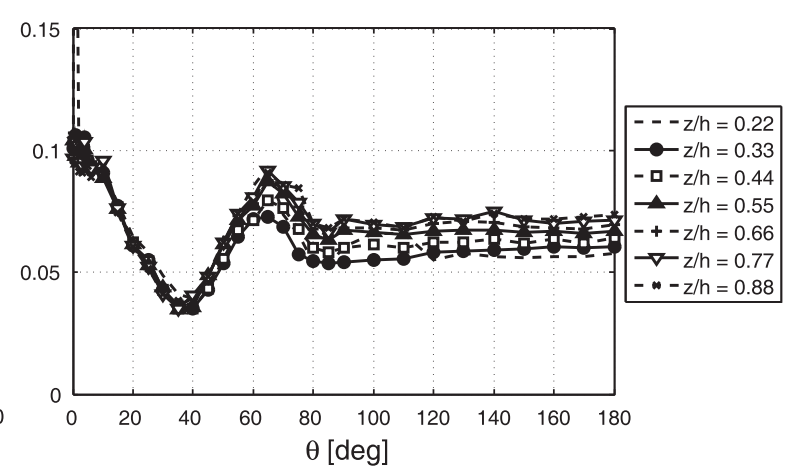

(b)

Fig. 8. Pressure field acting over the lateral surface measured for the freestream velocity $U_{\infty}=15 \mathrm{~m} / \mathrm{s}$ : (a) mean value of $C_{p}$; (b) standard deviation of $C_{p}$. 
generated by the flow passing over the free-end, whereas for the latter a reduced $C_{p}$ can be considered an effect of the velocity field induced by the small horse-shoe vortex that is probably present in that region. Moving to higher values of $\theta$, a lower $C_{p}$ is generally detected in proximity to the model free-end, also due to the induced upwash. Furthermore, the curve corresponding to $\theta=70^{\circ}$ suggests that the flow detachment over the lateral surface occurs at increasing $\theta$ moving from the model base towards the free-end; this delayed detachment for higher heights is most probably due to the upwash. Finally, for $\theta>80^{\circ}$ the mean pressure field remains fairly invariant for locations immersed into the separated wake.

The mean pressure field measured over the model free-end is reported in Fig. 10. Pressure suctions are more intense in proximity to the fore-portion of the free-end and then decrease moving towards the rear portion. The flow probably does not impinge over the free-end as no local peaks of $C_{p}$ are observed. However, slightly negative peaks located at $\theta \approx \pm 70^{\circ}$

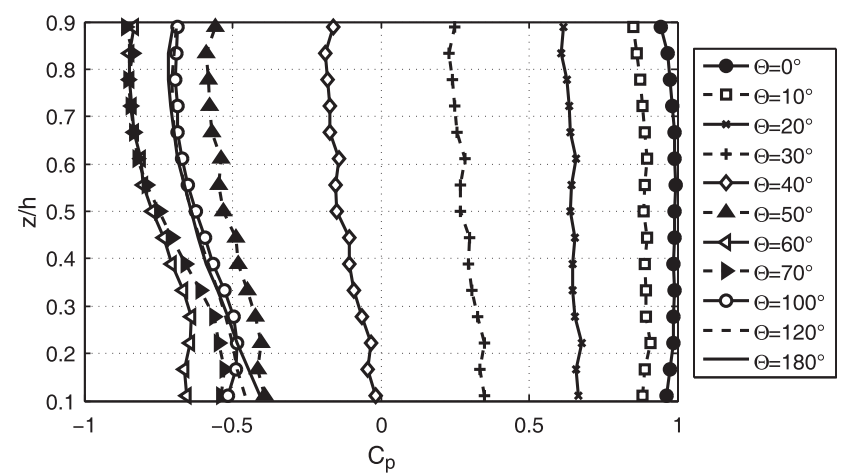

Fig. 9. Mean pressure field acting on the lateral surface as a function of $z / \mathrm{h}$ and measured at different $\theta$ at $U_{\infty}=15 \mathrm{~m} / \mathrm{s}$.

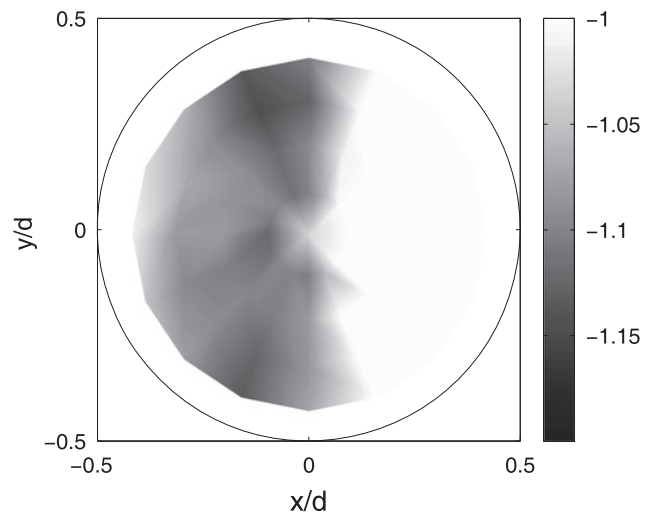

Fig. 10. Mean value of $C_{p}$ evaluated over the model free-end at $U_{\infty}=15 \mathrm{~m} / \mathrm{s}$.
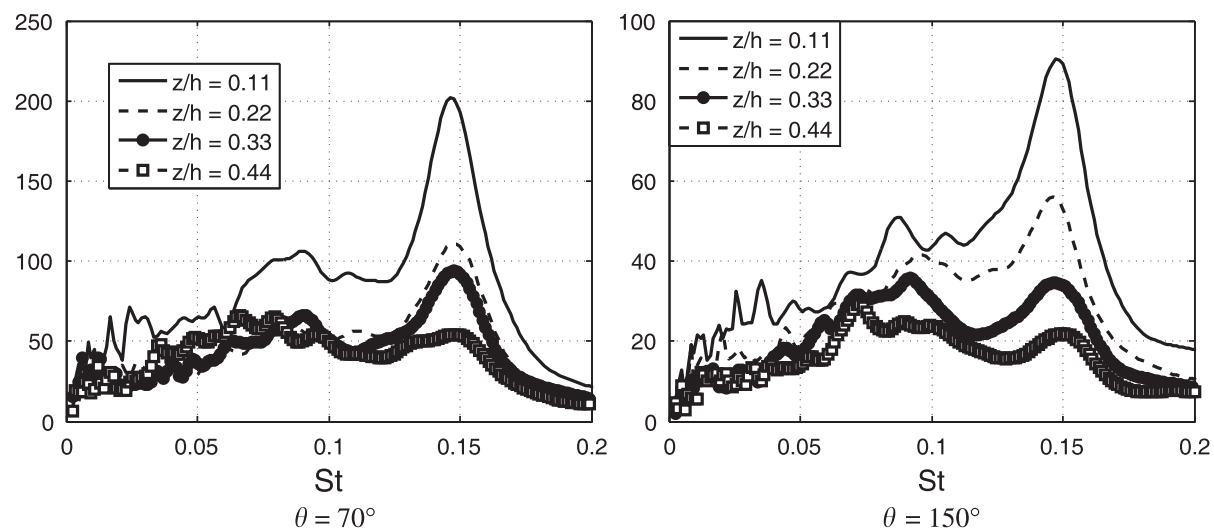

Fig. 11. Wavelet spectra of the pressure signals acquired over the lateral surface at several azimuthal locations and different heights at $U_{\infty}=25 \mathrm{~m} / \mathrm{s}$. 
and at a radial distance of about $19 \mathrm{~mm}$ from the model axis are observed, which are located roughly at the same positions where nuclei of vorticity structures were detected through oil flow visualizations by Roh and Park (2003) and Pattenden et al. (2005).

Subsequently, a spectral characterization of the pressure signals was carried out through wavelet transform; wavelet spectra of signals acquired over the lateral surface at several azimuthal positions, $\theta$, and different vertical positions are reported in Fig. 11. For azimuthal positions with $\theta<70^{\circ}$ no dominant spectral components are singled out. At $\theta \approx 70^{\circ}$, i.e. just before the flow separation, a dominant spectral contribution at $\mathrm{St} \approx 0.15$ is observed. Its fluctuating energy decreases rapidly with increasing height, and for $z / h>0.5$ it is not clearly detectable anymore. This feature indicates that a two-dimensional mechanism of vortex shedding takes place only for very low heights and that the flow in correspondence to the higher half-span of the model is dominated by the three-dimensional flow due to the presence of the free-end.

By increasing $\theta$ the fluctuating energy of the spectral component of interest is gradually reduced, up to $\theta \approx 180^{\circ}$ for which this spectral component is not detectable anymore.

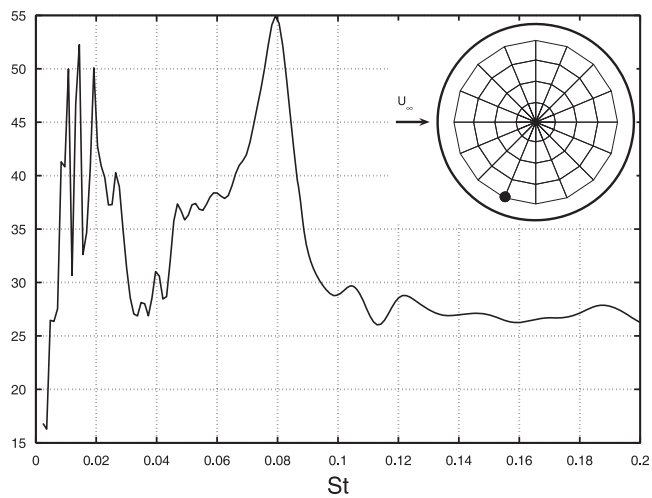

$r=25 \mathrm{~mm} ; \theta=292^{\circ}$

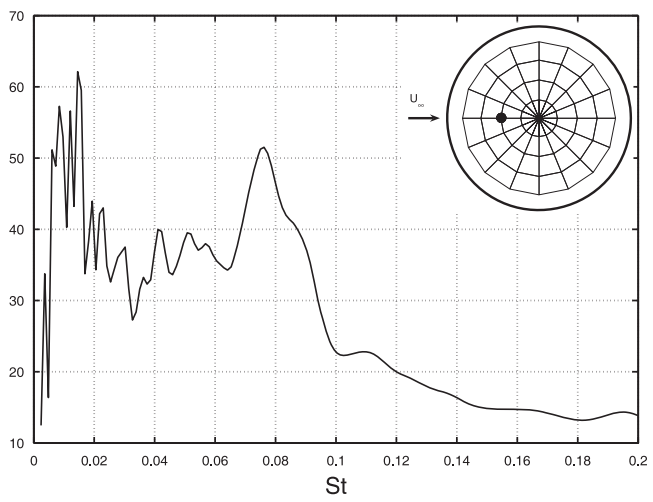

$r=12.5 \mathrm{~mm} ; \theta=0^{\circ}$

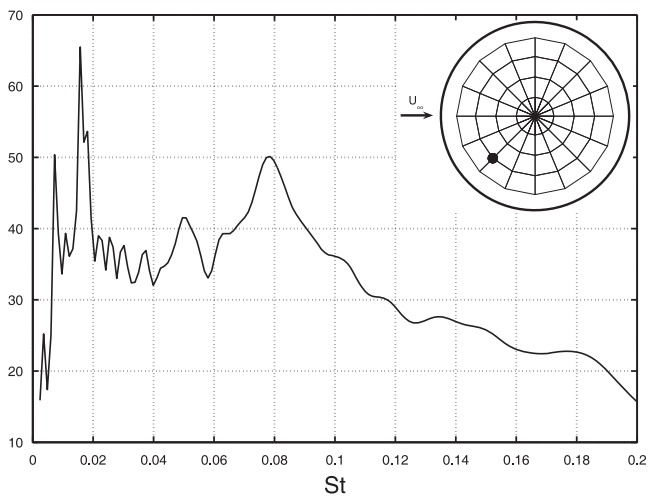

$r=19 \mathrm{~mm} ; \theta=315^{\circ}$

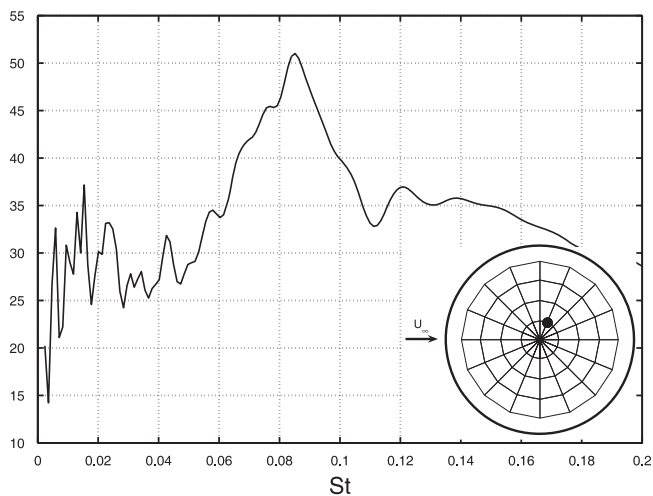

$r=6 \mathrm{~mm} ; \theta=112^{\circ}$

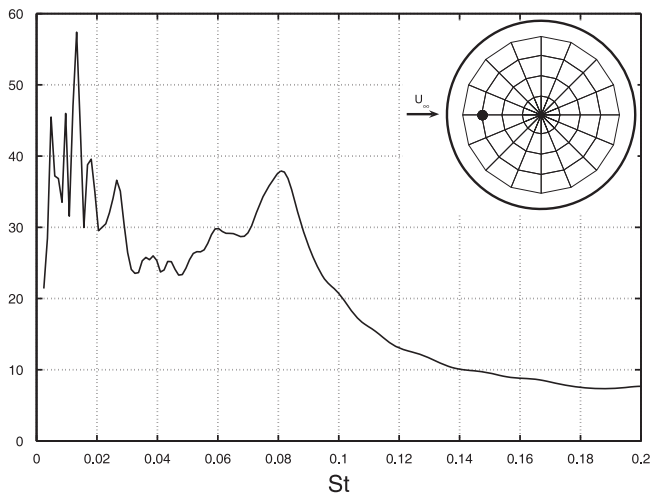

$r=19 \mathrm{~mm} ; \theta=0^{\circ}$

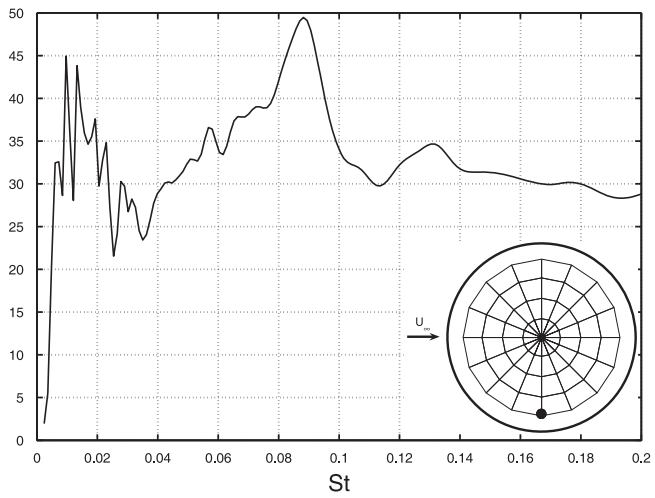

$r=25 \mathrm{~mm} ; \theta=270^{\circ}$

Fig. 12. Wavelet spectra of pressure signals acquired over the model free-end with a freestream velocity $U_{\infty}=25 \mathrm{~m} / \mathrm{s}$. Pressure taps are indicated through their azimuthal location and radial distance from the model axis. 
As for the free-end, in Fig. 12 it can be observed that the spectral component at St $\approx 0.15$ is not detected, as expected, because the alternate vortex shedding no longer dominates when the free-end is approached. However, a significant amount of fluctuating energy is observed for $S t \approx 0.07-0.08$, but it is hard to characterize it because of its high irregularity. This feature is in good agreement with what was found in Park and Lee (2000) where a dominant spectral component at St $\approx 0.07$ was found with a fluctuating energy that decreases with reducing $h / d$, even if it was not clearly detectable anymore already for a model with $h / d=6$.

\section{Conclusions}

The aim of the present work was characterizing the flow features of the wake generated from a circular cylinder with aspect-ratio 3 and placed vertically on a plane. Force measurements have been carried out by using a strain-gage balance at Reynolds numbers ranging in the interval $6 \times 10^{4} \leq \operatorname{Re} \leq 11 \times 10^{4}$. The mean drag coefficient, $C_{x}$, has been found to be roughly invariant by varying the test Reynolds numbers and a mean value $C_{x} \cong 0.81$ has been evaluated. As for the fluctuating forces, a dominant spectral component in the wavelet spectra has been detected only for the signals of the cross-flow force, with an average Strouhal number of 0.156 .

A spectral contribution with roughly the same Strouhal number is also present in the velocity signals acquired, through a hot-wire single-component anemometry, in proximity to the lateral wake boundary. However, its energy decreases by moving the probe horizontally away from the wake and vertically towards the free-end. Simultaneous measurements carried out at symmetrical locations with respect to the wake symmetry plane have shown that these fluctuations can confidently be ascribed to an alternate vortex shedding.

Subsequently, dynamic measurements of the pressure field acting on the lateral surface and the free-end of the model were carried out. From these measurements the spectral component connected to vortex shedding has been found to appear over the lateral surface with its maximum fluctuating energy at an azimuthal position just before the separation of the shear layers. The pressure fluctuations connected to vortex shedding then decrease by moving towards regions immersed in the separated wake. Furthermore, the energy connected to this spectral component decreases with increasing vertical coordinate and at about $50 \%$ of the model height an evident energy peak cannot be detected anymore. This indicates that the fluctuations connected with vortex shedding appear in the pressure field only for the lower half-span of the model, whereas for higher heights the upwash due to the presence of the free-end is probably the dominating flow feature.

From the spectral analysis of the pressure measurements carried out over the model free-end no evidence of the presence of the spectral component at $\mathrm{St} \approx 0.15$ was found, as expected. On the other hand, a significant fluctuating energy was observed at lower frequencies; however, an accurate characterization of a dominant spectral component was not achieved due to the high irregularity of the relevant fluctuations, which is most probably due to the low aspect-ratio of the considered model.

\section{References}

Ayoub, A., Karamcheti, K., 1982. An experiment on the flow past a finite circular-cylinder at high subcritical and supercritical Reynolds-numbers. Journal of Fluid Mechanics 118, 1-26.

Bearman, P.W., 2011. Circular cylinder wakes and vortex-induced vibrations. Journal of Fluids and Structures 27, 648-658.

Budair, M., Ayoub, A., Karamcheti, K., 1991. Frequency measurements in a finite cylinder wake at a subcritical Reynolds number. AIAA Journal 29, 2163-2168.

Buresti, G., Lombardi, G., 2003. Experimental analysis of the upper-wake flow field of finite cylinders with triangular and circular cross-section. In: Proceedings of the 16th AIMETA Congress of Theoretical and Applied Mechanics, Ferrara, CDrom.

Buresti, G., Lombardi, G., Bellazzini, J., 2004. On the analysis of fluctuating velocity signals through methods based on the wavelet and Hilbert transforms. Chaos, Solitons \& Fractals 20, 149-158.

E.S.D.U., 2005. Mean forces, pressures and flow flied velocities for circular cylindrical structures: single cylinder with two-dimensional flow. E.S.D.U. Item 80025.

Farivar, D., 1981. Turbulent uniform-flow around cylinders of finite length. AIAA Journal 19, 275-281.

Fox, T.A., Apelt, C.J., West, G.S., 1993. The aerodynamic disturbance caused by the free-ends of a circular cylinder immersed in a uniform flow. Journal of Wind Engineering and Industrial Aerodynamics 49, 389-400.

Fröhlich, J., Rodi, W., 2004. LES of the flow around a circular cylinder of finite height. International Journal of Heat and Fluid Flow 25, 537-548.

Griffith, M.D., Leontini, J., Thompson, M.C., Hourigan, K., 2011. Vortex shedding and three-dimensional behaviour of flow past a cylinder confined in a channel. Journal of Fluids and Structures 27, 855-860.

Khalak, A., Williamson, C.H.K., 1996. Dynamics of hydroelastic cylinder with very low mass and damping. Journal of Fluids and Structures $10,455-472$.

Kitagawa, T., Fujino, Y., Kimura, K., 1999. Effects of free-end condition on end-cell-induced vibration. Journal of Fluids and Structures 13, 499-518.

Kitagawa, T., Fujino, Y., Kimura, K., Mizuno, Y., 2002. Wind pressures measurement on end-cell-induced vibration of a cantilevered circular cylinder. Journal of Wind Engineering and Industrial Aerodynamics 90, 395-405.

Luo, S.C., Gan, T.L., Chew, Y.T., 1996. Uniform flow past one (or two in tandem) finite length circular cylinder(s). Journal of Wind Engineering and Industrial Aerodynamics 59, 69-93.

Okamoto, T., Yagita, M., 1973. The experimental investigation on the flow past a circular cylinder of finite length placed normal to the plane surface in a uniform stream. Bulletin of JSME 16, 805-814.

Okamoto, S., Sunabashiri, Y., 1992. Vortex shedding from a circular cylinder of finite length placed on a ground plane. Journal of Fluids Engineering 114 $512-521$.

Park, C.W., Lee, S.J., 2000. Free end effects on the near wake flow structure behind a finite circular cylinder. Journal of Wind Engineering and Industrial Aerodynamics $88,231-246$.

Park, C.W., Lee, S.J., 2002. Flow structure around a finite circular cylinder embedded in various atmospheric boundary layers. Fluid Dynamics Research 30 , 197-215. 
Park, C.W., Lee, S.J., 2004. Effects of free-end corner shape on flow structure around a finite cylinder. Journal of Fluids and Structures 19, 144-158.

Pattenden, R.J., Turnock, S.R., Zhang, X., 2005. Measurements of the flow over a low-aspect-ratio cylinder mounted on a ground plane. Experiments in Fluids 39, 10-21.

Roh, S.C., Park, S.O., 2003. Vortical flow over the free end surface of a finite circular cylinder mounted on a flat plate. Experiments in Fluids 34, 63-67. Sakamoto, H., Arie, M., 1983. Vortex shedding from a rectangular prism and a circular cylinder placed vertically in a turbulent boundary layer. Journal of Fluid Mechanics 126, 147-165.

Sakamoto, H., Oiwake, S., 1984. Fluctuating forces on a rectangular prism and a circular cylinder placed vertically in a turbulent boundary layer. Journal of Fluid Engineering 106, 160-166.

Stocks, N.G., Shaw, C.T., King, G.P., 1999. Energy distribution in modes in the wake of a finite-length cylinder before and after transition. Journal of Fluids and Structures $13,143-152$.

Sumner, D., Heseltine, J.L., Dansereau, O.J.P., 2004. Wake structure of a finite circular cylinder of small aspect ratio. Experiments in Fluids 37, 720-730. Suthon, P., Dalton, C., 2011. Streakline visualization of the structures in the near wake of a circular cylinder in sinusoidally oscillating flow. Journal of Fluids and Structures 27, 885-902.

Taniguchi, S., Sakamoto, H., Arie, M., 1981. Flow around circular cylinders of finite height placed vertically in turbulent boundary layers. Bulletin of JSME 24, 37-44.

Visscher, J., Pettersen, B., Andersson, H.I., 2011. Experimental study on wake behind tapered circular cylinders. Journal of Fluids and Structures 27, 1228-1237. 